\title{
Separation of 1,4-benzodiazepine Derivates by Micellar Electrokinetic Capillary Chromatography Using Cyclodextrines as Buffer Modifiers ${ }^{\dagger}$
}

\author{
Gabriel Hancu* and Árpád Gyéresi \\ Department of Pharmaceutical Chemistry, Faculty of Pharmacy, University of Medicine and Pharmacy Târgu Mureş, \\ Gh. Marinescu 38, 540000 Târgu Mureş, Romania
}

RECEIVED SEPTEMBER 30, 2010; REVISED NOVEMBER 15, 2010; ACCEPTED DECEMBER 3, 2010

\begin{abstract}
The applicability of micellar electrokinetic capillary chromatography (MEKC) for the separation of benzodiazepine (BZD) derivates with different structural characteristics has been studied. Efforts were first focused on the optimization of the analytical conditions, on the effects of buffer concentration, surfactant concentration and buffer $\mathrm{pH}$ on the separation. To manipulate selectivity of the separation $\beta$ cyclodextrin (CD) was added to the buffer solution, and urea was used to improve the solubility of the $\mathrm{CD}$ in water. The CD-modified MEKC separation of the eight BZDs was achieved within 15 minutes using 25 mmol dm${ }^{-3}$ natrium tetraborate- $50 \mathrm{mmol} \mathrm{dm}{ }^{-3}$ sodium dodecyl sulphate- $15 \mathrm{mmol} \mathrm{dm}{ }^{-3} \beta-\mathrm{CD}-2 \mathrm{~mol} \mathrm{dm}$ urea ( $\mathrm{pH} 9.3$ ) as the running buffer. The proposed separation method was evaluated on the basis of preci-
\end{abstract} sion, linearity and limit of detection. (doi: $10.5562 /$ cca1763)

Keywords: benzodiazepines, separation, capillary electrophoresis, micelles, cyclodextrines

\section{INTRODUCTION}

Benzodiazepines (BZDs) are the most widely used psychotropic drugs, especially for their anxiolityc effects, but some compounds are also used as sedatives, hypnotics, anticonvulsants, muscular relaxants or general anesthetics. ${ }^{1}$ To emphasize even more their importance we can mention the great number of officinal BZD derivates in the modern pharmacopoeias: 15 derivates in the $6^{\text {th }}$ edition of the European Pharmacopoeia - EPh6, ${ }^{2} 14$ derivates in the British Pharmacopoeia 2009 $\mathrm{BPh} 2009^{3}$ and 17 derivates and other 6 pharmaceutical formulations containing BZDs in the United States Pharmacopoeia 32 - USP32. ${ }^{4}$

The great therapeutic importance of these compounds is closely linked with their analytical aspects, so development of new methods of analysis is a permanent challenge and also a necessity.

Since its introduction, capillary electrophoresis (CE), nowadays an officinal method in EPh6, ${ }^{2}$ has shown great potential in the analysis of pharmaceutical compounds. Due to its speed of analysis, high separation efficiency and low solvent and sample consumption, $\mathrm{CE}$ is being regarded as an alternative or a complementary separation technique to the more frequently used high-performance liquid chromatography (HPLC). ${ }^{5}$
BZDs are mostly neutral from electrophoretic point of view and have almost similar hydrophobicities, ${ }^{6}$ hence a good separation by the conventional capillary zone electrophoresis (CZE), method based on the differences between the own electrophoretic mobilities of the analytes, cannot be expected. This problem can be solved by micellar electrokinetic capillary chromatography (MEKC), method that extends the applicability of $\mathrm{CE}$ to neutral molecules.

This method is based on the addition of a surfactant (sodium dodecyl sulphate - SDS) to the buffer solution above its critical micellar concentration (CMC). When the BZD sample is injected, it partitions itself between the buffer and micelle, therefore, the separation occurs due to the differences in the partition coefficient between the two phases, in a chromatography-like mode. $^{3,7}$

The anionic SDS micelles are electrostatically attracted towards the anode, but because of the electroosmotic flow (EOF) which is usually stronger than the electrophoretic migration, the anionic micelles will slowly migrate towards the cathode, in the direction of the detector. ${ }^{7,8}$

Our previous studies ${ }^{9,10}$ showed that in order to improve the separation of BZD we have to use additives (organic solvents - methanol, acetonitrile) to modify the

\footnotetext{
$\dagger$ Presented at the $10^{\text {th }}$ International Symposium and Summer School on Bioanalysis within the CEEPUS Network CII-HU-001004-0910, Zagreb, Croatia, July 2010.

* Author to whom correspondence should be addressed. (E-mail: g_hancu@yahoo.com)
} 


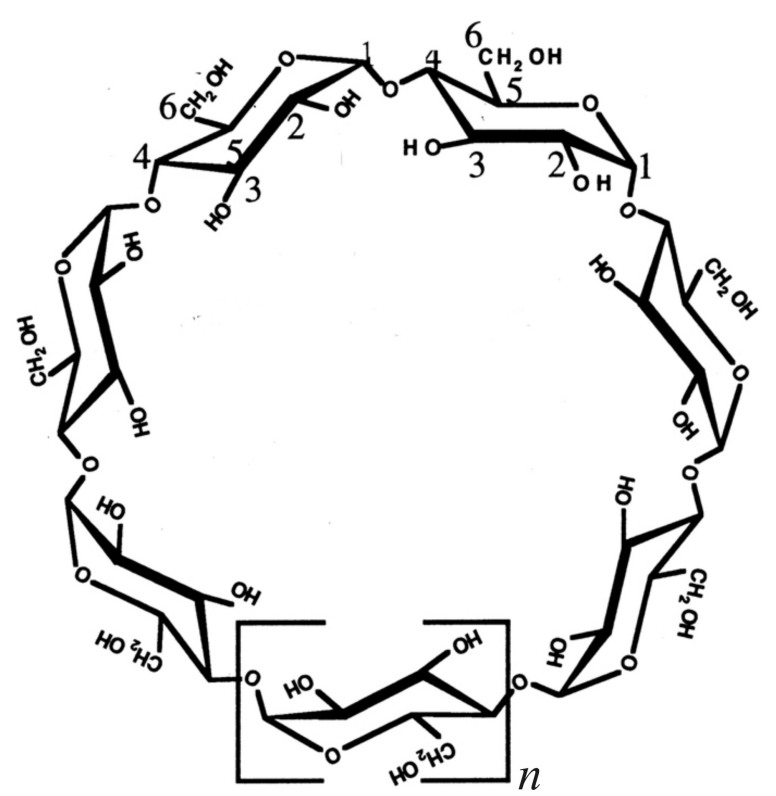

Figure 1. Chemical structure and schematic model of cyclodextrins: $\alpha-\mathrm{CD}(n-0), \beta-\mathrm{CD}(n-1)$ and $\gamma-\mathrm{CD}(n-2)$.

aqueous phase consequently manipulating selectivity. In general, addition of organic solvents reduces the electroosmotic velocity and, hence, expands the migration time window, improving selectivity of the separation. ${ }^{9,11,12}$

Another alternative would be the addition of cyclodextrines (CDs) to the buffer solution. CDs are cyclic oligosaccharides having an external hydrophilic surface and a hydrophobic cavity, in which they can include other compounds, which fit in their cavity by hydrophobic interaction. The most common and commercially available are the $\alpha-, \beta$-, and $\gamma$-CDs, formed by $\alpha-1,4-$ linked glucopyranose subunits, have the shape of a truncated cone and contain six, seven respectively eight subunits in their structure (Figure 1). ${ }^{13,14}$

In the present work 8 of the most frequently used BZD derivates were analyzed by means of MEKC. Our aim was not only the separation of the 8 BZD derivates but also the optimization of analytical conditions (efforts were focused on the study of the effect of buffer concentration, modifier concentration, additive concentration, buffer $\mathrm{pH}$ on the separation).

\section{EXPERIMENTAL}

We chose eight of the most frequently used BZD derivates, each having different structural characteristics: ${ }^{1,6}$ alprazolam, bromazepam, medazepam, nitrazepam (Labormed, Bucureşti, Romania), chlorazepate dipotassium, chlordiazepoxide, diazepam, oxazepam (Terapia, Cluj-Napoca, Romania) (Figure 2).

We used substances and reagents of analytical grade: natrium tetraborate, sodium dodecyl sulphate,

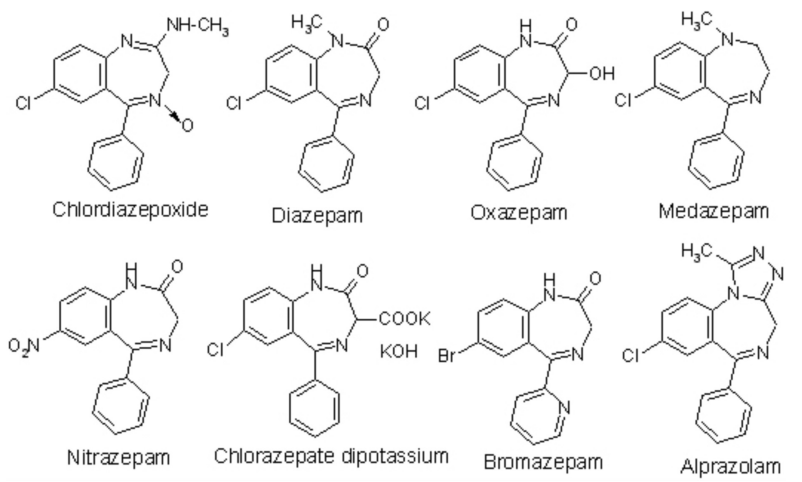

Figure 2. Chemical structure of the studied BZDs.

urea (Merck, Darmstadt, Germany), sodium hydroxide 0,1N (Fluka, Buchs, Switzerland), $\beta$-cyclodextrin (CD) (Cyclolab, Budapest, Hungary).

The CE instrument was an Agilent CE 6100 system. Separations were performed on polyimide-coated fused silica capillaries of $64.5 \mathrm{~cm}$ (effective length 56 $\mathrm{cm})$ X $50 \mu \mathrm{m}$ (Agilent, Waldbronn, Germany). The samples were introduced by hydrodynamic injection (50 mbar for 5 seconds) at the anodic end of the capillary. The applied voltage was $+25 \mathrm{kV}$. The generated current was kept below $200 \mu \mathrm{A}$. The temperature of the capillary holder was kept at $25{ }^{\circ} \mathrm{C}$. The detection was carried out by on-column photometric measurement at $214 \mathrm{~nm}$. The diode array detection (DAD) system made it possible to record the UV spectra at different points of the electropherogram. The electropherograms were recorded and processed by Chemstation software (Agilent, Waldbronn, Germany).

The sample solutions were prepared by dissolving the solid salts in methanol, and then by diluting it with water (1:1). Chlorazepate being used, as a potassium salt is not soluble in methanol, so in this case the solution was prepared by solubilization in water and dilution with methanol. The concentration of the stock solutions was $100 \mathrm{mg} / \mathrm{l}$. The capillaries were preconditioned with the buffer electrolyte for 5 minutes. After and the beginning of each daily work the capillaries were flushed with sodium hydroxide $0.1 \mathrm{~mol} \mathrm{dm}^{-3}$ (5 minutes) and distilled water ( 5 minutes) to remove all the components which may stick to the capillary walls. All the samples and buffers were filtered through a $0.45 \mu \mathrm{m}$ syringe filter and stored in a refrigerator at $+4{ }^{\circ} \mathrm{C}$.

\section{RESULTS AND DISCUSSION}

As we anticipated CZE was not suited for the separation of neutral substances like BZDs. All BZDs migrated very close the EOF, with the exception of chlorazepate dipotassium, which was used as a salt, so is a ionic analyte, consequently has its own electrophoretic mobility, and 
can be separated from the other BZDs by CZE using a buffer solution containing $25 \mathrm{mM}$ sodium tetraborate.

The higher the concentrations of electrolyte (borate ions), the later the migration times for each BZD, because the EOF decreases with an increase in ionic strength. In this work, the borate concentration was set at $25 \mathrm{mmol} \mathrm{dm}^{-3}$.

The migration times of the studied BZDs increased with increasing SDS concentration, due to the solubilization of the solute in the micellar phase. In this work, the SDS concentration was set at $50 \mathrm{mmol} \mathrm{dm}^{-3}$. Higher concentrations of surfactants $\left(>100 \mathrm{mmol} \mathrm{dm}^{-3}\right)$ resulted in relatively high viscosities and high currents, and should be avoided.

To decide the optimum $\mathrm{pH}$ value, we examined the value between 3.6 and 9.8, and found that the migration times had the tendency to increase at lower $\mathrm{pH}$, but had no marked effect over the $\mathrm{pH} 8$ to $10 .^{9,10}$

However using only SDS, the retention factors of BZDs are high, due to their high and comparable hydrophobicity. Therefore, these drugs migrate close to the SDS micelle and resolution is limited.

This problem was resolved by adding a neutral CD to the micellar solution. CDs are electrically neutral and have no electrophoretic mobility, consequently will not be incorporated in the micelle because of the hydrophilic nature of the outside. The migration velocity and hence the migration times of BZDs depends on the partitioning of the solute between the $\mathrm{CD}$ and the micelle (Figure 3). ${ }^{8,15,16}$

This technique is limited to solutes, which can fit into the CD cavity. The ratio of the solute incorporated into the micelle depends on its hydrophobicity but the inclusion complex formation of the solute with $\mathrm{CD}$ depends on the concordance of the solute molecular size with the CD cavity diameter. ${ }^{13,15}$

Using a molecular modulation program, Chem3D (CambridgeSoft), we followed the molecular complexation of BZDs with CDs. Having a rigid structure with a benzene ring condesated to a diazepinic one the complexation process for BZDs is difficult and far from perfect.

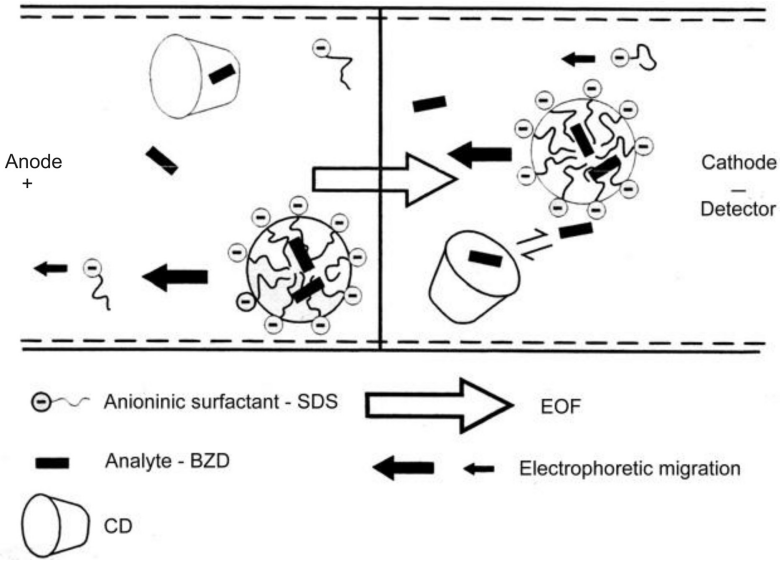

Figure 3. Schematic of the separation principle of CD-MEKC.

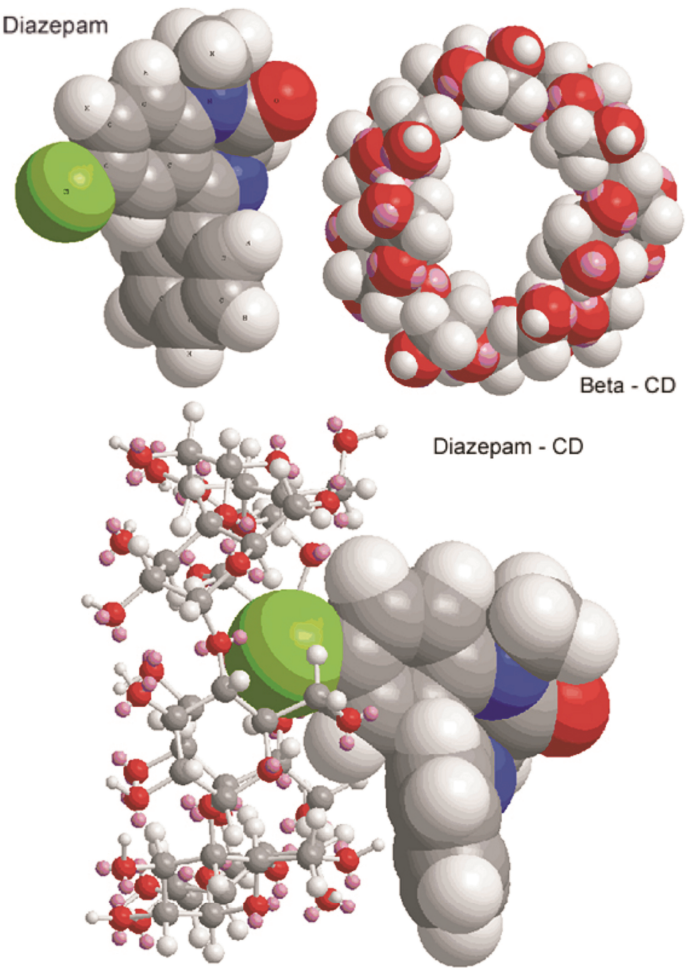

Figure 4. Complexation of Diazepam with $\beta-\mathrm{CD}$.

BZD can't fit in the cavity of $\alpha-C D$, but fit even if just partially in cavities of $\beta$ - respectively $\gamma$-CD (Figure 4).

Among various $\mathrm{CDs}, \gamma-\mathrm{CD}$ in general is considered to be most effective in MEKC separations, ${ }^{14,15}$ but in this work we chose $\beta-C D$ arguably the most frequently used $\mathrm{CD}$ for the complexation of chemical compounds of pharmaceutical interest.

A decrease in the EOF and a shortening of the migration time of the BZDs with increasing CD concentration was observed (Figure 5). High concentration of CDs may lead to an increase of the solubilization in the CD ca-

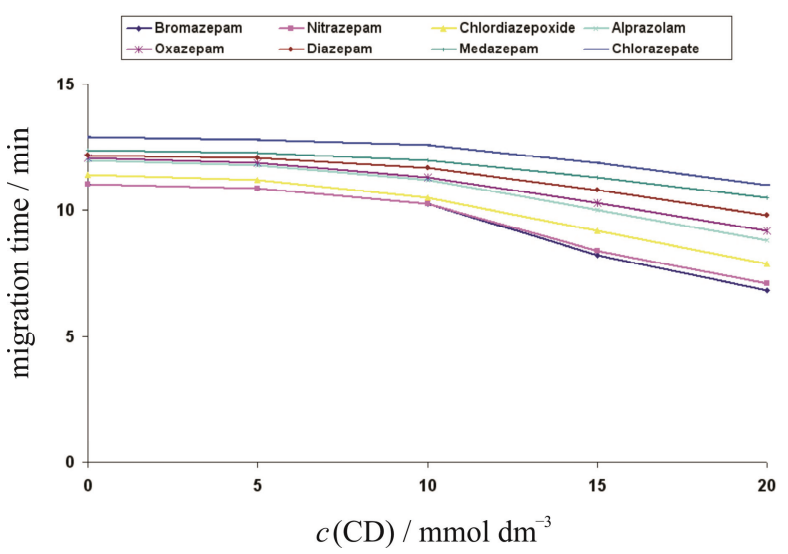

Figure 5. Influence of $\mathrm{CD}$ concentration on the separation of BZDs (separation conditions: capillary $50 \mathrm{~cm} \times 50 \mu \mathrm{m}$ I.D, buffer electrolyte $25 \mathrm{mM}$ borate $+50 \mathrm{mM}$ SDS, pH 9.3, applied voltage $+25 \mathrm{kV}$, detection UV $214 \mathrm{~nm}$, concentration of each BZD $10 \mu \mathrm{g} / \mathrm{ml}$ ). 


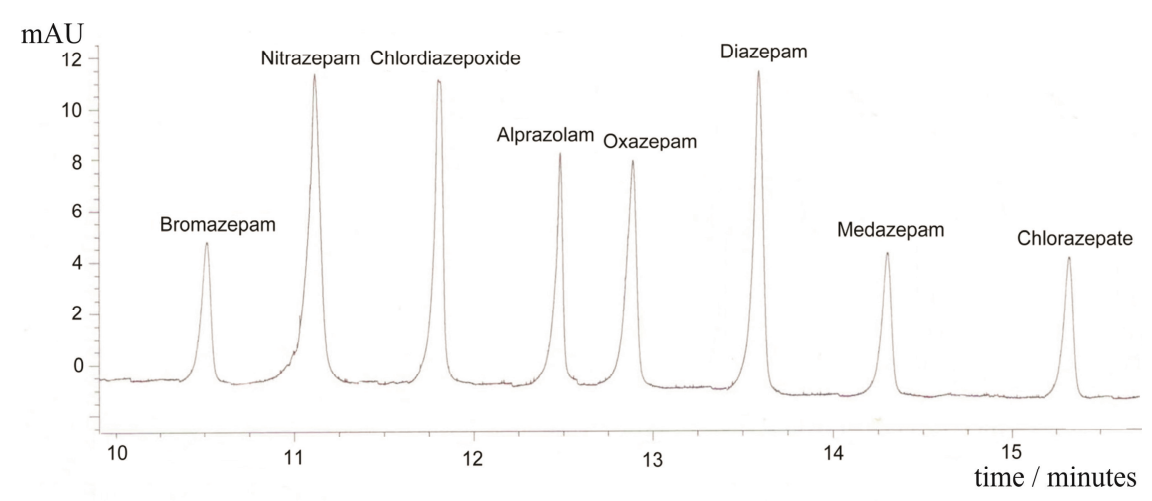

Figure 6. Electropherogram of a mixture of BZDs using a a buffer solution containing $25 \mathrm{mM}$ natrium tetrabotate, $50 \mathrm{mM}$ SDS, $15 \mathrm{mM} \beta$-CD and $2 \mathrm{M}$ urea $(\mathrm{pH}-9.3)$ (separation conditions: capillary $50 \mathrm{~cm} \times 50 \mu \mathrm{m} \mathrm{I.D}$, applied voltage $+25 \mathrm{kV}$, detection UV $214 \mathrm{~nm}$, concentration of each BZD $10 \mu \mathrm{g} / \mathrm{ml})$.

vity, and migration of the solutes with the EOF. ${ }^{15}$ In this work, the CD concentration was set at $15 \mathrm{mmol} \mathrm{dm}^{-3}$.

The solubility of $\beta-C D$ in water is relatively low. Addition of a high concentration of urea $\left(2 \mathrm{~mol} \mathrm{dm}^{-3}\right)$ was used to increase solubility. Urea slightly reduces the electrosmotic velocity and considerably reduces the migration velocity of the micelle, improving the separation of highly hydrophobic compounds such as BZDs.

We obtained the best electropherograms using a

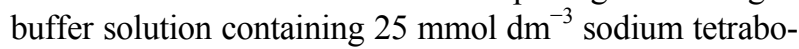
rate, $50 \mathrm{mmol} \mathrm{dm}^{-3} \mathrm{SDS}, 15 \mathrm{mmol} \mathrm{dm}{ }^{-3} \beta-\mathrm{CD}$ and $2 \mathrm{~mol}$ $\mathrm{dm}^{-3}$ urea ( $\mathrm{pH}$ 9.3) (Figure 6), when we managed the separation of the 8 BZDs in less then 15 minutes. The separation occurred in the following order: bromazepam, nitrazepam, chlordiazepoxide, alprazolam, oxazepam, diazepam, medazepam and chlorazepate dipotassium.

The elution order should agree with the tendency of the BZDs to form inclusion complexes with the CD in the presence of SDS. A solute having a shorter migration time is more strongly included in the $\mathrm{CD}$ cavity than a solute having a longer migration time.

It is well established that oxazepam and chlorazepate dipotassium are optically active, having a chiral center in the $\mathrm{C}_{3}$ position. ${ }^{1}$ However pure enantiomers are difficult to isolate, because they are quickly racemized in aqueous medium. It is interesting that peak splitting didn't occurred when we used $\beta-C D$ as buffer additive, but separation of the two enantiomers can be solved using hydroxypropyl- $\beta-\mathrm{CD}$ (HP- $\beta-\mathrm{CD})$ as additive, because of the different affinities displayed by the selector molecules towards the two enantiomers. ${ }^{17}$

The analytical performance of the optimized method was evaluated on the basis of precision (by calculating regional standard deviation- RSD for the migration time and peak area), linearity and limit of detection (LOD).

The calculation of LODs was based on a signal-tonoise ratio of 3 . Very similar migration time and peak area were obtained for ten repeated measurements of the eight compounds which proves a good precision of the method (Table 1).

The individual linear regression equations for each BZD were calculated according to six concentrations and three replicates per concentrations. The correlation coefficient ranged between 0.983 (alprazolam) and 0.993 (diazepam). The analytical signal shows a linear dependence on the analyte concentration, in the concentration domain between $5-100 \mu \mathrm{g} / \mathrm{ml}$.

Table 1. Analytical parameters of the separation of BZD derivates

\begin{tabular}{lcccc}
\hline Benzodiazepines & $\begin{array}{c}\text { Migration time / } \\
\text { min }\end{array}$ & $\begin{array}{c}\text { Mobility / } \\
\mathrm{cm}^{2} \mathrm{kV}^{-1} \mathrm{~min}^{-1}\end{array}$ & $\begin{array}{c}\text { LOD / } \\
\mu \mathrm{g} \mathrm{ml}\end{array}$ & $\begin{array}{c}\text { RSD / \% } \\
\text { migration time }\end{array}$ \\
\hline Bromazepam & 10.50 & -10.35 & 1.42 & $\begin{array}{c}\text { RSD / \% } \\
\text { peak area }\end{array}$ \\
Nitrazepam & 11.15 & -11.11 & 0.61 & 0.21 \\
Chlordiazepoxide & 11.85 & -11.87 & 0.63 & 0.20 \\
Alprazolam & 12.45 & -12.46 & 0.98 & 0.21 \\
Oxazepam & 12.90 & -12.78 & 0.92 & 0.18 \\
Diazepam & 13.60 & -13.36 & 0.58 & 0.15 \\
Medazepam & 14.40 & -14.03 & 1.38 & 0.28 \\
Chlorazepate & 15.30 & -14.62 & 1.51 & 0.58 \\
\hline
\end{tabular}

signal $/$ noise ratio $=3$.

$c=100 \mu \mathrm{g} / \mathrm{ml}, n=10$. 


\section{CONCLUSIONS}

In summary, we studied in detail the $\mathrm{CE}$ conditions for the analysis of BZD derivates.

MEKC can separate both ionic and neutral substances while CZE separates only ionic substances. The separation principle of MEKC is based on the partition of the solute between the micelle and water, while in CZE is based on the differential electrophoretic mobility of the analytes.

The use of additives to modify the aqueous phase is very effective in manipulating selectivity of the separation. The addition of $\mathrm{CD}$ reduces the distribution coefficient and enables the separation of highly hydrophobic analytes, which otherwise would be almost completely incorporated into the micelle.

The selectivity and resolution of the method can be also controlled and manipulated by varying the buffer concentration, surfactant concentration, buffer $\mathrm{pH}$ or CD concentration.

The optimal running buffer was $25 \mathrm{mmol} \mathrm{dm}{ }^{-3}$ natrium tetraborate- $50 \mathrm{mmol} \mathrm{dm}$ sodium dodecyl sulphate- 15 mmol dm${ }^{-3} \beta-C D-2$ mol dm${ }^{-3}$ urea (pH 9.3), the running time was around 15 minutes and the elution order was: bromazepam, nitrazepam, chlordiazepoxide, alprazolam, oxazepam, diazepam, medazepam, chlorazepate dipotassium.

CD-MEKC proved an efficient tool for the separation of the studied BZD derivates and probably for other substances with similar chemical structures. The methods can find practical application in the determination of BZD derivates from biological fluids.

Acknowledgements. This study was supported by the CEEPUS program (CII-HU-0010 network).

\section{REFERENCES}

1. Wilson\&Gisvold Textbook of Organic Medicinal and Pharmaceutical Chemistry, $11^{\text {th }}$ edition, Lippincott Williams\&Wilkins, Baltimore, 2004, 488-492.

2. European Pharmacopoeia, $6^{\text {th }}$ edition, Council of Europe, Strasbourg, 2007.

3. British Pharmacopoeia 2009, Her Majesty’s Stationary Office, London, 2009.

4. United States Pharmacopoeia 32, United States Pharmacopoeia Convention Inc, Rockville, 2009.

5. Encyclopedia of Chromatography, $3^{\text {rd }}$ edition, volume 1, CRC Press Taylor\&Francis Group, Boca Raton, 2010, 339-360.

6. H. Schutz, Benzodiazepines, basic data, analytical methods, pharmacokinetics and comprehensive literature, Springer Verlag, Berlin, 1989.

7. K. D. Altria, Capillary Electrophoresis Guidebook: Principles, Operation and Applications, Humana Press, Totowa, New Jersey, 1998.

8. S. Terabe, Micellar Electrokinetic Chromatography, Beckham Catalog, 1998.

9. G. Hancu, A. Gáspár, and Á. Gyéresi, J Biochem. Biophys. Meth. 69 (2007) 251-259.

10. G. Hancu, Á. Gyéresi, and A. Gáspár, Acta Pharm. Hung. 77 (2007) 123-131.

11. M. Tomita and T. Okoyuma, J. Chromatogr. B 678 (1996) 331-337.

12. M. Imazawa and Y. Hatanaka, J. Pharm. Biomed. Anal. 15 (1997) 1503-1508.

13. B. Tőkés, L. Ferencz, P. Buchwald, G. Donáth-Nagy, S. Vancea, N. Sánta, and E. L. Kis, J Biochem. Biophys. Meth. 70 (2007) 1276-1282.

14. H. T. L. Luong and A. L. Nguyen, J. Chromatogr. A 792 (1997) 431-444.

15. M. F. Renou-Gonnord and K. David, J. Chromatogr. A 735 (1996) 249-261.

16. S. C. Bishop, M. Lerch, and B. R. Mc Cord, Foren. Sci. Int. 141 (2004) 7-15.

17. G. Hancu, A. Gáspár, and Á. Gyéresi, Farmacia, LVI(4) (2008), 381-388. 